\title{
Hoarding Disorder in a Patient with Schizophrenia and Mild Cognitive Impairment: A Case Report
}

\author{
Case Report
}

Volume 2 Issue 1- 2021

\begin{abstract}
Author Details
Muhammad Aadil ${ }^{1}$, Joseph Elfar ${ }^{2}$, Muhammad Atif Ameer ${ }^{3 *}$ and Muhammad Ismail Khalid Yousaf ${ }^{4}$

${ }^{1}$ Department of Psychiatry, Rutgers New Jersey Medical School, USA

${ }^{2}$ Department of Psychiatry, Winchester Medical Center, USA

${ }^{3}$ Department of Neurology, University of Nebraska Medical Center, USA

${ }^{4}$ Department of Neurology, University of Louisville, USA

*Corresponding author

Muhammad Atif Ameer, Department of Neurological Sciences, University of Nebraska Medical Center, Movement Disorders Division, 988440 Nebraska Medical Center, Omaha, NE, USA
\end{abstract}

Article History

Received: April 01, 2021 Accepted: April 06, 2021 Published: April 08, 2021

\begin{abstract}
:
Hoarding disorder is a psychiatric pathology where the patient has difficulty discarding items, has severe anxiety related to the idea of discarding objects, and excessive possession leading to clutter, further intensifying distress and impairment of function. We report a case of a 60 years old male patient with schizophrenia and mild cognitive impairment who developed a hoarding disorder. Because of the severity of the symptoms, the patient required hospitalization. Conjugation of the pharmacotherapeutic and psychotherapeutic approaches helped the patient manage his symptoms.
\end{abstract}

Keywords: Hoarding disorder; Schizophrenia; Cognitive Impairment; Psychiatry; Obsessive-compulsive disorder; Cognitivebehavioral therapy; Anti-psychotic therapy

\begin{abstract}
Abbreviations: HD: Hoarding Disorder; OCD: ObsessiveCompulsive Disorder; DSM: Diagnostic and Statistical Manual of Mental Disorders, $5^{\text {th }}$ edition

\section{Introduction}

Hoarding disorder is defined today as a psychiatric pathology where patients have trouble discarding items causing these items to build up and congest the living space of the patient and limit their functionality [1].

Hoarding disorder was first included in the Diagnostic and Statistical Manual of Mental Disorders under the diagnostic criteria of obsessivecompulsive personality disorder. Clinical hoarding was considered a secondary behavioral phenomenon to some disorders such as dementia, residual schizophrenia, eating disorders, brain injury, and others. Extreme hoarding was considered a type of ObsessiveCompulsive Disorder (OCD) and described in the fourth edition of the Diagnostic and Statistical Manual of Mental Disorders [1,2]. Hoarding disorder is included in the fifth edition of the Diagnostic
\end{abstract}

and Statistical Manual of Mental Disorders (DSM-5), and it has its own criteria for diagnosis [3].

An important distinguishing feature between Hoarding Disorder (HD) and OCD is the intrusiveness of the thoughts to hoard. In OCD, the thoughts are intrusive, repetitive, and ego-dystonic, which causes stress when the patient does not act. Whereas in HD, the thoughts about hoarding do not cause stress, the stress comes from the clutter build-up that makes a space uninhabitable or dangerous to live in [4]. Surveys primarily in Europe and the United States have estimated the point prevalence of clinically significant hoarding to be approximately $2-6 \%$ among adults and $2 \%$ among adolescents $[1,5]$. A study done in England using the strict DSM-5 criteria found a prevalence of $1.5 \%$ in men and women [6]. Treatment for hoarding disorder is separated into psychosocial interventions and pharmacotherapy. Clinical trials have demonstrated efficacy in reducing hoarding symptoms using this cognitive-behavioral model of therapy for hoarding [7]. Research into pharmacotherapy for hoarding disorder has investigated several drugs such as serotonin reuptake inhibitors [8], methylphenidate [9], and atomoxetine [10]. However, these uncontrolled trials and case 
series do not reveal strong evidence of their use for reducing hoarding behavior in hoarding disorder.

\section{Case Report}

We present a case of a 60-year-old African American man with a past psychiatric history of schizophrenia and mild cognitive impairment. He was brought to the hospital from his nursing facility after a suspected suicide attempt and dangerous behaviors. The staff found him in his room with a towel wrapped around his neck and head with several burners, and hot plates turned on. The staff also reported that the patient was collecting knives, towels, clothes, and other items in his room. Post-admission to the hospital, the patient continued exhibiting hoarding behavior. The patient would collect random items like orange peels, old used towels, socks, disposable hospital gowns, styrofoam cups, plastic water pitchers, deodorant, and lotion. He also started collecting multiple pillows, blankets, and bed sheets in his room. The patient would never discard the items by himself, and the patient was resistant to throwing things away. When asked why he kept so many items, the patient would often rationalize, saying that he might need them in the future. The thoughts to keep the possessions were not intrusive, repetitive, or ego-dystonic. When asked when this hoarding behavior began, the patient said it started 1-2 years ago, he began collecting many things in his room, and it has been getting progressively worse. The patient's mental status examination showed a mildly disoriented man who was calm, pleasant with intact cognition, linear, and goal-directed thought process. Despite the patient's insistence that he did not want to end his life, collateral collected from his nursing home revealed that they had a high suspicion for a suicide attempt as the patient kept many knives, scissors, and burners in his room.

A treatment plan for the patient was designed. The plan addressed the patient's hoarding issues by educating the patient and having him exposed to discarding objects that he was hoarding. The treatment plan also addressed the patient's schizophrenia and his major depressive disorder. He was initially started on risperidone $1 \mathrm{mg}$ oral at night, risperidone $0.5 \mathrm{mg}$ oral in the morning, Bupropion ER $150 \mathrm{mg}$ oral, Gabapentin $300 \mathrm{mg}$ oral daily, Fluoxetine $20 \mathrm{mg}$ oral daily. The patient was also on milieu therapy, which is psychotherapy that controls the patient's environment to prevent them from harming themselves. By the end of the patient's stay, he was taken off of fluoxetine, increased on his risperidone to $2 \mathrm{mg}$ twice daily, Bupropion ER $300 \mathrm{mg}$ for his depression, gabapentin $300 \mathrm{mg}$ oral for anxiety. The patient continued to show significant improvement in his behavior problem during his stay in the hospital.

\section{Discussion}

We presented a case of hoarding disorder in a patient with chronic schizophrenia and major depressive disorder in remission. Given the patient's limited social support, the patient was treated primarily with pharmacotherapy and psychotherapy during his stay in the hospital. The patient met the DSM-V criteria for hoarding disorder. The patient was seen collecting random objects, showed severe distress discarding them. The hoarding behavior was resulting in impairment of social functioning and was not an outcome of any other mental disorder or medical condition. The behavior of collecting objects, excessive anxiety, avoidance of discarding them mimicked obsessive-compulsive disorder. However, it was differentiated from OCD because of the lack of any intrusive or compulsive thoughts or images that lead to that behavior. Unlike OCD, the desire to collect objects was more egosyntonic.

Hoarding disorder mostly begins at an early age, has a stable chronic progressive course with some reports of waxing and waning pattern [1]. While some studies have found an increased prevalence of HD in the elderly population, others reported no association between age and development of HD. There is a reported correlation of onset of hoarding disorder after a severe life stressor and associated with lower neurocognitive functioning [1]. In our case, the patient started noticing the behavior at the age of 59 years. He had a history of bilateral foot amputation and lost his son a year ago before the onset of the symptoms. The Montreal Cognitive Assessment testing was used to screen for neurocognitive dysfunction, and it showed mild dysfunction (Score 21/30). It is also plausible that the symptoms of the hoarding disorder do not progress with age, but the decline of neurocognition results in difficulty dealing with the outcome of the behavior, further impairing organization and structure, such as in this case.

HD was primarily being treated with cognitive behavioral therapy, which includes motivational interviewing, psychoeducation, and classic cognitive techniques to target dysfunctional beliefs and behavior. A study conducted in 2014 showed promising results of Cognitive-Behavioral Therapy (CBT) alone targeting exposure function and memory. Regarding pharmacotherapy- Selective serotonin reuptake inhibitors have been suggested for the treatment of HD. However, most studies are open-ended with limited subjects. A study conducted in 2014 used Paroxetine and showed a 36\% decrease in UCLA Hoarding Severity Scale (UHSS) in 12 weeks of treatment [8]. In our case, we used Risperidone $1 \mathrm{mg}$ BID and Bupropion 150 $\mathrm{mg}$, which was later increased to $300 \mathrm{mg}$ to manage schizophrenia and depression. No studies are available found to compare these pharmacotherapeutic agents along with CBT in their effectiveness to manage HD symptoms.

Overall, a conjugation of pharmacotherapeutic and psychotherapeutic approaches helped our patient to manage his HD. More studies are required to compare the effectiveness of different pharmacotherapeutic drugs along with CBT to help patients manage their symptoms, especially in older age with multiple comorbidities.

\section{Disclosure}

The authors declare that the research was conducted in the absence of any commercial or financial relationships that could be construed as a potential conflict of interest.

\section{References}

1. Pertusa A, Frost RO, Fullana MA, Samuels J, Steketee G, et al. (2010) Refining the diagnostic boundaries of compulsive hoarding: a critical review. Clinical psychology review 30(4): 371-386.

2. Mataix-Cols D, Frost RO, Pertusa A, Clark LA, Saxena S, et al. (2010) Hoarding disorder: A new diagnosis for DSM-V? Depression and anxiety 27(6): 556-572

3. Association AP (2013) Paraphilic disorders. Diagnostic and statistical manual of mental disorders 5: 685-686.

4. Morgado P, Freitas D, Bessa JM, Sousa N, Cerqueira JJ (2013) Perceived stress in obsessive-compulsive disorder is related with obsessive but not compulsive symptoms. Front Psychiatry 4: 21 .

5. Ivanov VZ, Mataix-Cols D, Serlachius E, Lichtenstein P, Anckarsäter $\mathrm{H}$, et al. (2013) Prevalence, comorbidity and heritability of hoarding symptoms in adolescence: a population based twin study in 15-year olds. PloS one 8(7): e69140.

6. Nordsletten AE, Reichenberg A, Hatch SL, de la Cruz LF, Pertusa A, et al (2013) Epidemiology of hoarding disorder. Br J Psychiatry 203(6): 445452 .

7. Frost RO, Hartl TL (1996) A cognitive-behavioral model of compulsive hoarding. Behav Res Ther 34(4): 341-350.

8. Saxena S, Sumner J (2014) Venlafaxine extended-release treatment of hoarding disorder. Int Clin Psychopharmacol 29(5): 266.

9. Rodriguez CI, Bender Jr J, Morrison S, Mehendru R, Tolin D, et al. 
(2013) Does extended release methylphenidate help adults with hoarding disorder? A case series. Journal of clinical psychopharmacology 33(3): 444 .
10. Grassi G, Micheli L, Di Cesare Mannelli L, Compagno E, Righi L, et al. (2016) Atomoxetine for hoarding disorder: A pre-clinical and clinical investigation. J Psychiatr Res 83: 240-248. 